\title{
Prognostic significance of high triglyceride and apolipoprotein B levels in patients with stage III and high-risk stage II colorectal cancer undergoing curative surgery
}

\author{
XIU-QING CHEN ${ }^{1,2}$, PEI-WEN WU ${ }^{1,2}$, DONG-HUI LIU ${ }^{1,2}$, \\ SUN-JIE YAN ${ }^{1,2}$, XI-MEI SHEN ${ }^{1,2^{*}}$ and LI-YONG YANG ${ }^{1,2^{*}}$ \\ ${ }^{1}$ Department of Endocrinology, The First Affiliated Hospital of Fujian Medical University; \\ ${ }^{2}$ Diabetes Research Institute of Fujian Province, Fuzhou, Fujian 350005, P.R. China
}

Received July 7, 2019; Accepted April 7, 2020

DOI: $10.3892 / \mathrm{ol} .2020 .11617$

\begin{abstract}
Although epidemiologic studies suggest that dyslipidemia increases the risk of colorectal cancer (CRC), the prognostic value of blood lipid and apolipoprotein levels in CRC remains unclear. The aim of the present study was to investigate the impact of blood lipid and apolipoprotein levels on the prognosis of patients with stage III and high-risk stage II CRC undergoing curative surgery. Preoperative levels of total cholesterol, triglycerides (TG), high-density lipoprotein, low-density lipoprotein, very-low-density lipoprotein, apolipoprotein A1 and apolipoprotein B (APO-B) in patients with CRC undergoing surgery were evaluated. The cut-off values of these factors were determined by the maximal $\mathrm{x}^{2}$ method and were used to classify patients into two prognostic groups: Poor and good prognosis groups. The patients' prognostic values were assessed using the Kaplan-Meier curve and Cox regression analysis. In addition, the impact of these parameters on the prognosis and their predictive accuracy were evaluated using nomograms and Harrell's concordance index,
\end{abstract}

Correspondence to: Professor Li-yong Yang or Professor Xi-mei Shen, Department of Endocrinology, The First Affiliated Hospital of Fujian Medical University, 20 Cha Zhong Road Fuzhou, Fujian 350005, P.R. China

E-mail: liyongy@medmail.com.cn

E-mail: niaoshe2006@163.com

${ }^{*}$ Contributed equally

Abbreviations: APO-A1, apolipoprotein-A1; APO-B, apolipoprotein B; $\mathrm{BMI}$, body mass index; CI, confidence interval; CEA, carcinoembryonic antigen; CRC, colorectal cancer; DFS, disease-free survival; OS, overall survival; DM, diabetes mellitus; DT; differentiation status; HDL, high-density lipoprotein; HR, hazard ratio; LDL; low-density lipoprotein; TC, total cholesterol; TG, triglyceride; TNM, Tumor-Node-Metastasis; VLDL, very low-density lipoprotein

Key words: colorectal cancer, lipids, survival, triglycerides, apolipoprotein B respectively. In total, 246 patients were included in this evaluation. Based on the cut-off points for TG $(1.53 \mathrm{mmol} / \mathrm{l}$ in men and $1.58 \mathrm{mmol} / \mathrm{l}$ in women) and APO-B $(0.73 \mathrm{mmol} / \mathrm{l}$ in men and women), the present study determined that both TG and APO-B were predictors of disease-free survival (DFS) and overall survival (OS). Multivariate analysis demonstrated that high TG (men, $\geq 1.53 \mathrm{mmol} / \mathrm{l}$; women, $\geq 1.58 \mathrm{mmol} / \mathrm{l}$ ) and high APO-B ( $\geq 0.73 \mathrm{mmol} / \mathrm{l})$ levels were significantly associated with decreased DFS and OS. Nomograms that included values for TG and APO-B levels demonstrated higher predictive accuracy compared with that of nomograms without these values. These results indicated that TG and APO-B levels may be good independent prognostic biomarkers after radical CRC surgery. Therefore, adjusting these parameters to moderate levels may be beneficial.

\section{Introduction}

Colorectal cancer (CRC) is the third most common cancer worldwide, with 1.8 million new cases and 881,000 deaths recorded in 2018 (1). CRC is also the second leading cause of cancer-related deaths (1). The overall 5 -year survival is only $65.2 \%$, and the stage-specific 5-year survival is 93.2 for stage I, 82.5 for stage II, 59.5 for stage III and $8.1 \%$ for stage IV in USA (2).

Recurrence following resection is a major problem in CRC, which results in poor prognosis, particularly in patients with stage III and high-risk stage II CRC. Currently, cancer prognosis depends mainly on the pathological stage at the time of diagnosis. However, patients with the same Tumor-Node-Metastasis (TNM) stage still have different prognoses (3).

In addition to staging prognosis, assessment of clinical biomarkers is an optimal strategy to identify patients with higher risks of recurrence and low survival (4). This distinction is helpful for patient management, optimization of current treatment options, and early detection of recurrence (5). Studies have reported that the age, sex, tumor location, grade, inflammatory markers and number of involved lymph nodes are prognostic factors of CRC (6).

Blood lipid markers are also associated with survival outcomes in patients with certain malignant tumors, including prostate, breast, gastrointestinal, cervical and lung 
cancers (7-11). However, the association between blood lipid levels and the prognosis of CRC has not been well studied, and the results of the studies conducted thus far are inconsistent. One study demonstrated that dyslipidemia, including high triglyceride (TG) levels and low high-density lipoprotein (HDL) cholesterol levels, was independently associated with improved overall and recurrence-free survival of patients with colon cancer (12). By contrast, a recent prospective study by Peng et al (13) that involved 1,318 patients with CRC reported that patients with dyslipidemia exhibited a significantly shorter median survival duration compared with those without dyslipidemia. Although patients with stage III and high-risk stage II CRC primarily have a poor prognosis after radical surgery, previous studies did not stratify patients by stage $(2,12-16)$. Furthermore, there are limited studies that have investigated the role of apolipoprotein levels in CRC prognosis. Thus, the current study aimed to investigate the association between lipid and lipoprotein levels and the prognosis of patients with stage III and high-risk stage II CRC.

\section{Materials and methods}

Patient selection. This retrospective study included patients diagnosed with CRC between June 2008 and September 2011 in the Department of Colorectal Surgery of the First Affiliated Hospital of Fujian Medical University in Fujian (Fujian, China). A total of 246 patients (134 male and 112 female) were enrolled in this study. The mean age of the patients was 60.56 \pm 13.23 (range, 21-84) years. The patients exhibited no distant metastases or local recurrence and underwent surgical resection. The TNM stages (17) of stage III and high-risk stage II CRC were confirmed by pathological examination. Among these patients, $56.8 \%$ of patients with right-sided colon cancer and $69.2 \%$ of patients with left-sided colon cancer received chemotherapy after surgical resection. Neo-adjuvant chemoradiation was administered to $83.2 \%$ of patients with tumors located in the rectal area.

The inclusion criteria were as follows: i) Stage III or high-risk stage II CRC; and ii) complete clinical data, including age, sex, height, weight, history of diabetes mellitus (DM), history of hypertension, smoking status, alcohol intake, cancer site, tumor stage, histological class, differentiation and the levels of carcinoembryonic antigen (CEA), total cholesterol (TC), triglyceride (TG), high-density lipoprotein cholesterol (HDL), low-density lipoprotein cholesterol (LDL), very-low-density lipoprotein cholesterol (VLDL), apolipoprotein-A1 (APO-A1) and apolipoprotein B (APO-B). The exclusion criteria were as follows: i) Treatment with lipid-lowering agents or metformin; ii) presence of infections, serious liver and kidney dysfunction, severe heart disease or other serious illnesses; iii) history of other malignancies; iv) incomplete preoperative laboratory data; or v) use of anti-inflammatory medications before surgery.

Patients with high-risk stage II CRC were defined as those who had poor prognosis with the following characteristics: T4 (stage IIB, IIC), poor histological differentiation (grades 3/4, excluding high microsatellite instability) (18), vascular and nerve infiltration, intestinal obstruction, tumor site perforation, positive or unclear margins, inadequate margins and $<12$ lymph nodes sent for examination.
The present study was reviewed and approved by the Ethics Committee of The First Affiliated Hospital of Fujian Medical University (Fujian, China), and written informed consent was obtained from all subjects.

Data collection. Blood levels of CEA, TC, TG, HDL, LDL, VLDL, APO-A1 and APO-B were measured up to 7 days before the surgery using standard methods. Patients were not allowed to consume meals with high-fat content before blood tests to reflect the true level of blood lipids. Data on clinical parameters, including age at CRC diagnosis, sex, height, weight, body mass index (BMI), history of DM, history of hypertension, smoking status, alcohol intake, cancer site, TNM classification, histological type, differentiation status, perineural invasion, vascular tumor thrombus and intestinal obstruction were collected from the medical records. Tumor staging for CRC in this study was based on the 7th edition of the American Joint Committee on Cancer TNM classification (17). BMI was classified as underweight $\left(<18.5 \mathrm{~kg} / \mathrm{m}^{2}\right)$, normal weight $\left(18.5-23.9 \mathrm{~kg} / \mathrm{m}^{2}\right)$, overweight $\left(24.0-27.9 \mathrm{~kg} / \mathrm{m}^{2}\right)$ and obese $\left(\geq 28 \mathrm{~kg} / \mathrm{m}^{2}\right)$ according to the diagnostic criteria in China (19). Smoking status was defined as smoking $\geq 10$ cigarettes per day at the time of CRC diagnosis. Alcohol intake was defined as the consumption of at least one alcoholic drink per week at the time of CRC diagnosis.

Follow-up. Follow-up was conducted every 3-6 months for the first 2 years after resection, every 6 months for the next 3 years and annually thereafter. Follow-up procedures included colonoscopy, computed tomography and CEA tests. Local recurrence and distant metastasis from CRC were identified by endoscopy, tissue pathological examination or imaging analysis. The last follow-up was performed on May 1, 2018.

Statistical analysis. The primary endpoint was disease-free survival (DFS), and the secondary endpoint was overall survival (OS). DFS was defined as the time from the date of surgery to the date of local tumor recurrence and/or distant metastases or the date of the last follow-up. OS was defined as the time from the date of surgery to the date of death or the date of the last follow-up. The optimal cut-off values of TC, TG, HDL, LDL, VLDL, LDL/HDL ratio, APO-A1 and APO-B were determined using the maximal $\mathrm{x}^{2}$ method to best classify patients into two prognostic groups: Poor and good. The R MaxStat package (https://CRAN.R-project. org/package=maxstat) in $\mathrm{R}$ version 3.5.2 software (https:// cran.r-project.org) was used for this analysis (20). The Kaplan-Meier method was used to establish the effect of each variable on DFS, and log-rank tests were used to compare the survival curves. Univariate and multivariate analyses using the Cox proportional hazards model were also performed to identify the prognostic impact of clinical parameters and blood lipid index on DFS and OS. Significant variables in univariate analysis $(\mathrm{P}<0.1)$ were entered into regression models with increasing complexity, and significance was assessed using analysis of variance followed by a Bonferroni post hoc test. Data were represented as hazard ratios (HRs) and 95\% confidence intervals (CIs). A predictive nomogram of 1-, 3and 5-year CRC mortality was established based on clinical and clinicopathological parameters. The variables from Cox 


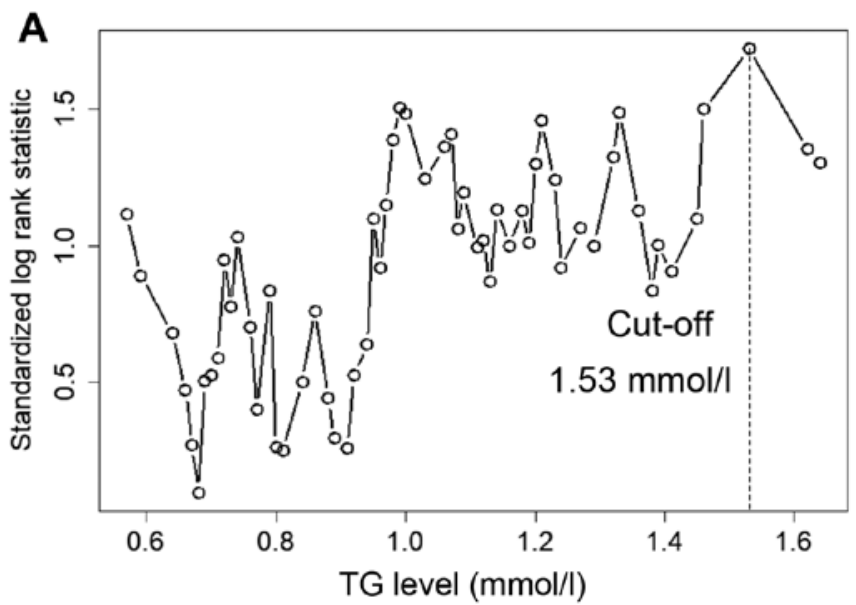

B

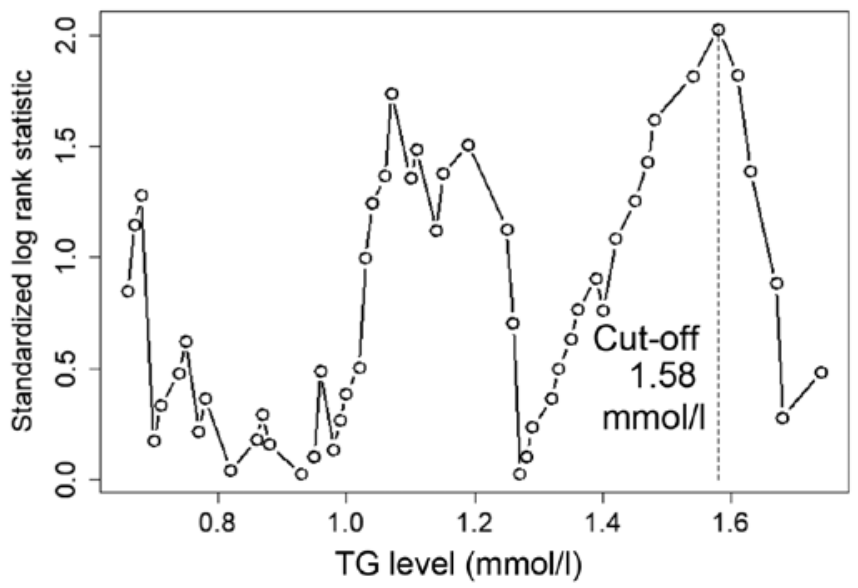

Figure 1. Cut-off value for TG. (A and B) The maximum difference in distant metastasis-free survival was achieved when the TG level was $1.53 \mathrm{mmol} / \mathrm{l}$ in (A) men and $1.58 \mathrm{mmol} / \mathrm{l}$ in (B) women. TG, triglycerides.

multivariate analysis were included in the nomogram analysis. The nomogram was implemented by the regression modeling strategy (RMS) package (http://biostat.mc.vanderbilt.edu/ wiki/Main/RmS) in $\mathrm{R}$ version 3.5.2 software (Institute for Statistics and Mathematics). The concordance index was used to evaluate the predictive accuracy of the nomogram. All statistical analyses were performed using SPSS 19.0 (SPSS, Inc.) and $\mathrm{R}$ version 3.5.2 software. $\mathrm{P}<0.05$ was considered to indicate a statistically significant difference

\section{Results}

Optimal cut-off values of lipid indices by sex. The optimal cut-off values for predicting DFS in men were determined to be as follows: TC, $3.30 \mathrm{mmol} / \mathrm{l}$; TG, $1.53 \mathrm{mmol} / \mathrm{l}$; HDL, $0.98 \mathrm{mmol} / \mathrm{l}$; LDL, $1.91 \mathrm{mmol} / \mathrm{l}$; VLDL, $0.20 \mathrm{mmol} / \mathrm{l}$; LDL/HDL, 2.09; APO-A1, $1.05 \mathrm{~g} / 1$; and APO-B, and $0.73 \mathrm{~g} / \mathrm{l}$. The optimal cut-off values for predicting DFS in women were as follows: TC, $5.23 \mathrm{mmol} / \mathrm{l}$; TG, $1.58 \mathrm{mmol} / \mathrm{l}$; HDL, $1.18 \mathrm{mmol} / \mathrm{l}$; LDL, $1.91 \mathrm{mmol} / \mathrm{l}$; VLDL, $0.46 \mathrm{mmol} / \mathrm{l}$; LDL/HDL, $1.35 \mathrm{mmol} / 1$; APO-A1, $1.14 \mathrm{~g} / \mathrm{l}$; and APO-B, $0.73 \mathrm{~g} / 1$ (Figs. 1 and 2; Table I). The Kaplan-Meier analysis demonstrated that high TG and APO-B levels were significantly associated with poor DFS time (Fig. 3; Table I).
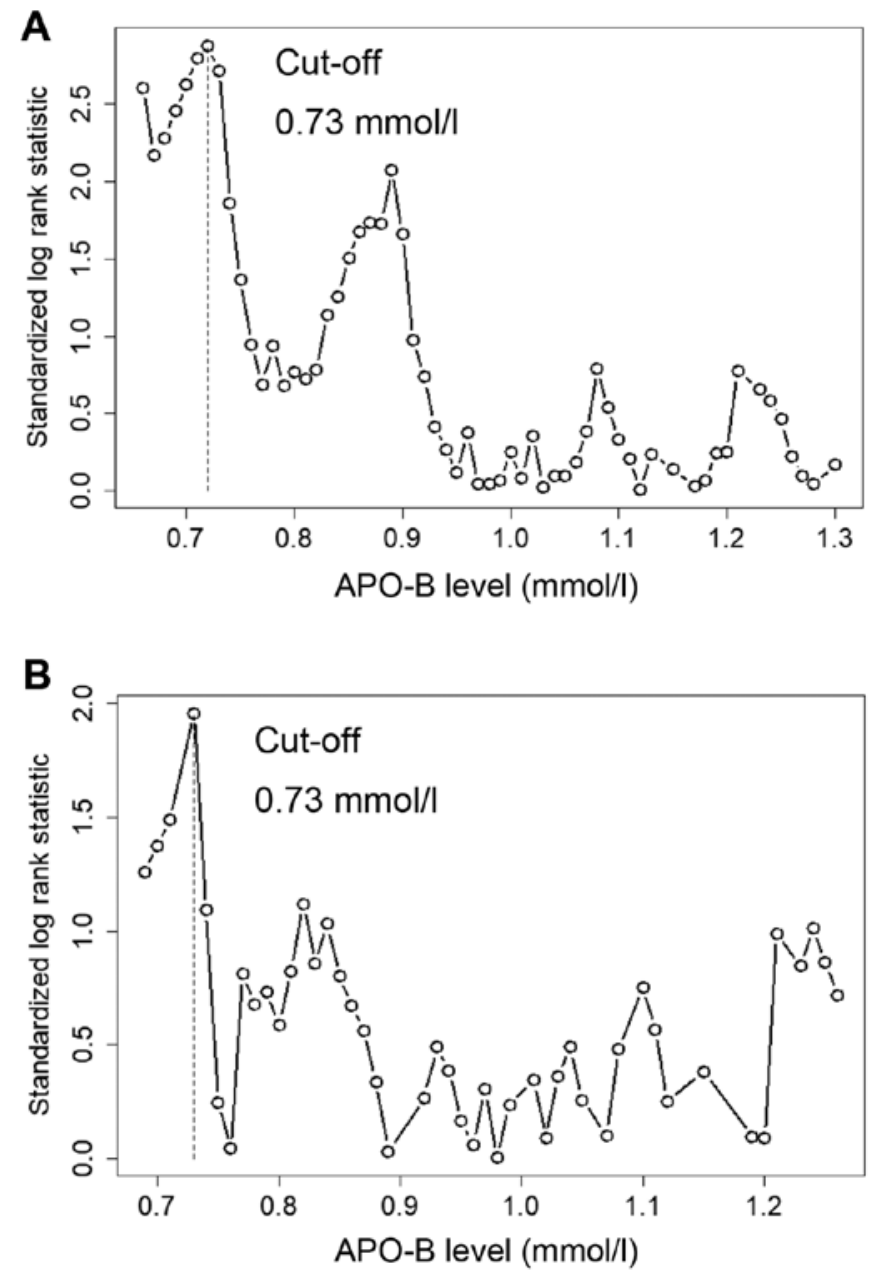

Figure 2. Cut-off value for APO-B. (A and B) The maximum difference in distant metastasis-free survival was observed at the APO-B level of $0.73 \mathrm{mmol} / 1$ in (A) men and (B) women. APO-B, apolipoprotein B.

Patient characteristics. A total of 246 patients were included in this study. Of these, 44 (17.9\%) had right-sided colon cancer, 65 (26.4\%) had left-sided colon cancer, and $137(55.7 \%)$ had rectal cancer. The baseline clinical and laboratory characteristics are listed in Table II. The median follow-up duration was 74 (range, 6-114) months. The median follow-up duration for patients who were alive at the end of this study was 95 months (range, 79-114). At the end of the study, 60 patients $(24.4 \%)$ had distant metastasis that mainly involved the lungs (43.3\%) and the liver (33.3\%). Local recurrence occurred in $13(5.3 \%)$ cases.

Univariate and multivariate survival analysis. Univariate survival analysis was used to study the associations of sex, age at diagnosis, history of DM, history of hypertension, smoking, alcohol intake, BMI, histological type, TNM stage, TG, APO-B, CEA, differentiation, perineural invasion, vascular tumor thrombus and intestinal obstruction with DFS and OS. The results of the univariate analysis demonstrated that TG (men $\geq 1.53 \mathrm{mmol} / 1$, women $\geq 1.58 \mathrm{mmol} / \mathrm{l})$, APO-B ( $\geq 0.73 \mathrm{mmol} / \mathrm{l})$, TNM stage, tumor location, perineural invasion and poor differentiation were identified as significant prognostic factors for DFS (Table III). TG, APO-B, TNM stage, tumor location and perineural invasion were 
Table I. Optimal cut-off values of the lipid indices by sex.

\begin{tabular}{lccccc}
\hline & & & \multicolumn{2}{c}{ Cut-off } \\
\cline { 4 - 5 } Index & Reference range & $\begin{array}{c}\text { No. of patients in the } \\
\text { normal range, } \mathrm{n}(\%)\end{array}$ & Male & Female & P-value \\
\hline $\mathrm{TC}, \mathrm{mmol} / \mathrm{l}$ & $3.60-5.69$ & $177(71.95)$ & 3.3 & 5.23 & 0.113 \\
$\mathrm{TG}, \mathrm{mmol} / \mathrm{l}$ & $0.34-1.70$ & $220(89.43)$ & 1.53 & 1.58 & $0.027^{\mathrm{a}}$ \\
$\mathrm{HDL}, \mathrm{mmol} / \mathrm{l}$ & $>1.04$ & $160(65.04)$ & 0.98 & 1.18 & 0.643 \\
$\mathrm{LDL}, \mathrm{mmol} / \mathrm{l}$ & $<3.64$ & $212(86.18)$ & 1.91 & 1.91 & 0.123 \\
VLDL, $\mathrm{mmol} / \mathrm{l}$ & $<0.78$ & $242(98.37)$ & 0.20 & 0.46 & 0.112 \\
LDL/HDL & $1.2-1.6$ & $194(78.86)$ & 2.09 & 1.35 & 0.544 \\
APOA1, g/l & $0.6-1.1$ & $181(73.58)$ & 1.05 & 1.14 & 0.071 \\
APO-B, $\mathrm{g} / \mathrm{l}$ & & & 0.73 & 0.73 & $0.036^{\mathrm{a}}$ \\
\hline
\end{tabular}

${ }^{\mathrm{a}} \mathrm{P}<0.05$. TC, total cholesterol; TG, triglyceride; HDL, high-density lipoprotein; LDL, low-density lipoprotein; VLDL, very low-density lipoprotein; APO-A1, apolipoprotein-A1; APO-B, apolipoprotein B.

identified as significant prognostic factors for OS (Table III). Variables with $\mathrm{P}<0.1$ in the univariate Cox regression analysis were used in the multivariate analysis based on forward stepwise selection. CEA and BMI were also analyzed in multivariate analysis (Table III).

In the multivariate Cox analysis, high TG levels (HR, 1.987; 95\% CI, 1.057-3.737), high APO-B levels (HR, 2.920; 95\% CI, 1.031-8.276), advanced TNM stage (HR, 3.258; 95\% CI, 1.606-6.608) and perineural invasion (HR, 2.891; 95\% CI, 1.573-5.312) were identified as independent prognostic factors for DFS in patients with CRC (Table III). High TG levels (HR, 2.374; 95\% CI, 1.318-4.275), high APO-B levels (HR, 2.425; 95\% CI, 1.019-5.775), advanced TNM stage (HR, 2.772; 95\% CI, 1.485-5.177) and perineural invasion (HR, 2.963; 95\% CI, 1.641-5.353) were identified as independent prognostic factors for OS in patients with CRC (Table III).

Nomogram. To assess the prognostic values of TG and APO-B for DFS and OS in patients with CRC, nomograms were constructed (Fig. 4). The variables from Cox multivariate analysis, including TG, APO-B, TNM stage, tumor location, perineural invasion, differentiation, CEA and BMI, were incorporated into the nomograms. The concordance index of the nomogram that included TG and APO-B values were 0.754 for DFS and 0.768 for OS, whereas the concordance index of the nomogram without TG and APO-B values were 0.732 for DFS and 0.726 for OS. These outcomes indicated that the concordance index of the nomogram involving TG and APO-B values may be improved compared with that of the nomogram without these values in predicting the clinical outcomes of CRC patients.

\section{Discussion}

In the present study, the effects of blood lipid and apolipoprotein on the prognosis of radical CRC were investigated. The results of the present study demonstrated that high TG and APO-B levels were independent prognostic factors for poor DFS and OS in patients with stage III and high-risk stage II CRC.
To the best of our knowledge, this is the first study to describe the relationship between APO-B levels and CRC prognosis. The patients were into two groups using a cut-off value of $0.73 \mathrm{mmol} / \mathrm{l}$ based on the $\mathrm{R}$ MaxStat method. Multivariate analysis and the nomogram demonstrated that a baseline high serum APO-B level was a predictor of poor DFS and OS in patients with CRC. APO-B is the main apolipoprotein of chylomicrons, VLDL, intermediate-density lipoprotein and LDL particles (21). Thus, it is responsible for transporting fat molecules to all peripheral tissues. APO-B is also the major structural protein for atherogenic APO-B-containing lipoproteins (22). The epidemiological evidence for the association between APO-B levels and CRC prognosis has not been confirmed, and the conclusions concerning this association are controversial. Borgquist et al (23) conducted a prospective cohort study that included 28,098 individuals with an average follow-up of 14.3 years and reported that the levels of APO-B were positively associated with the risk of CRC and lung cancer. Another study with an average follow-up of 15.6 years conducted by Katzke et al (24) revealed that high levels of circulating APO-B were positively associated with the risk of breast cancer. By contrast, in a study on tumor prognosis, Chen et al (25) reported that high levels of APO-B were beneficial for the OS of patients with lung cancer. In a retrospective study involving 1,201 patients with gastric cancer, those with high APO-B/APO-A1 ratios had a shorter OS (26).

The present study demonstrated that patients with high TG levels (men, $\geq 1.53 \mathrm{mmol} / 1$; women, $\geq 1.58 \mathrm{mmol} / \mathrm{l}$ ) had worse DFS and OS. TGs constitute a part of the lipid profile in the human body and are the major component of chylomicrons and VLDL (27). TGs are formed from fatty acids and stored in the adipose tissue, and they are the main form of energy storage and circulation (28). TGs are also involved in protein transport and serve as energy sources obtained from dietary fat (27). Patients with obesity or DM often present with high TG levels (29). In addition, a high TG level is one of the diagnostic criteria for the metabolic syndrome (27). Due to the known associations of obesity, DM and the metabolic syndrome with CRC progression, a growing number of studies have focused on the relationship between TG levels and 
A

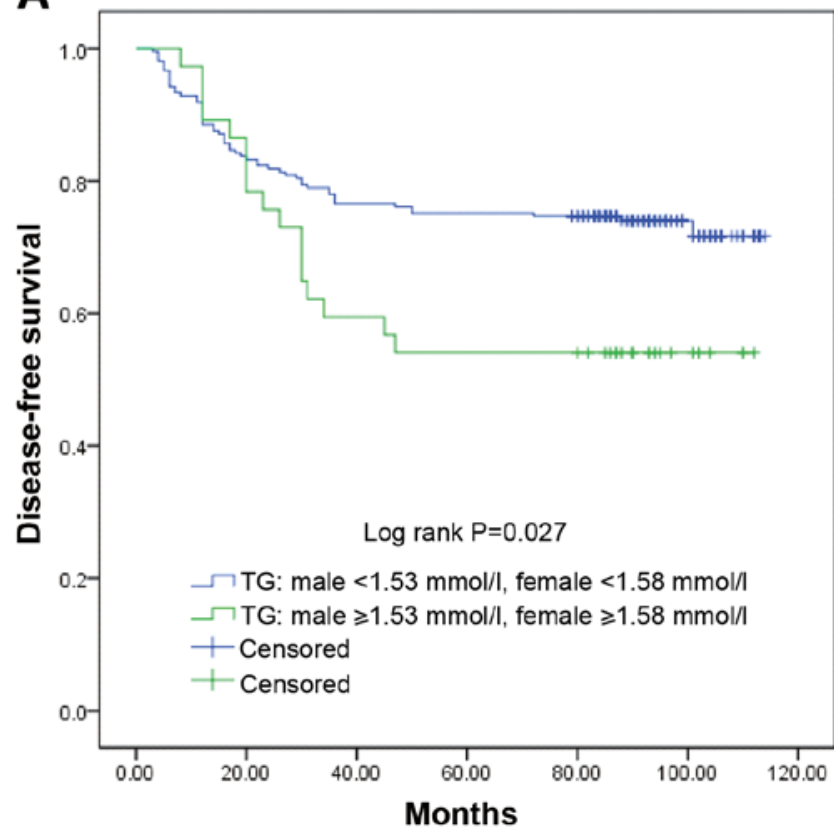

B

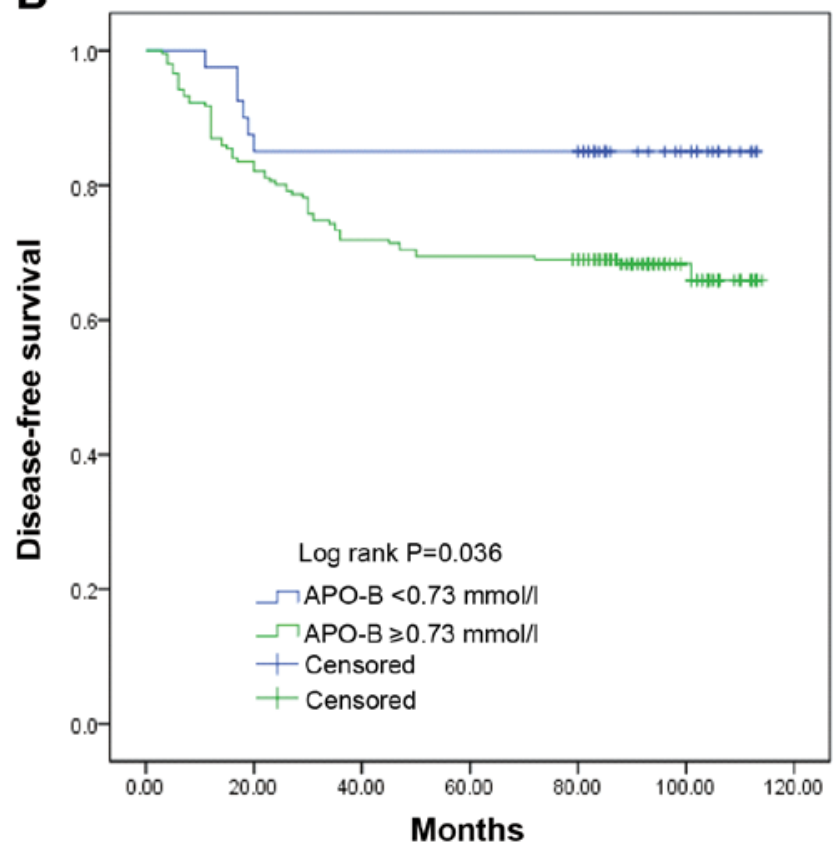

Figure 3. Kaplan-Meier analysis of the effects of each variable on DFS. (A) The DFS curves of patients with different TG levels. (B) The DFS curves of patients with different APO-B levels. The log-rank test was used to compare survival curves. DFS, disease-free survival; TG, triglycerides; APO-B, apolipoprotein B.

CRC (30,31). A meta-analysis of 16 studies indicated that high levels of TG increased the overall risk of cancer by $20 \%$ (32). The results of another meta-analysis of 17 studies, which assessed the association between CRC risk and TG levels, demonstrated that high TG levels increased the overall CRC risk by $6 \%$; however, this increase was not statistically significant (33). A large prospective study that included 514,097 participants with an average follow-up of 13.4 years indicated that high levels of serum TG were associated with a significant two-fold increase in the risk of colon cancer in men but not in women (34).
Table II. Demographic and clinical characteristics of the study population $(\mathrm{n}=246)$.

\begin{tabular}{|c|c|c|c|}
\hline Characteristics & Value & $\%$ & $\begin{array}{c}\text { Median } \\
(\min , \max )\end{array}$ \\
\hline Number of patients & 246 & & \\
\hline Age ( $\geq 60$ years) & 139 & 56.5 & \\
\hline Sex (male) & 134 & 54.5 & \\
\hline History of DM & 40 & 16.3 & \\
\hline History of HP & 61 & 24.8 & \\
\hline Smoking & 33 & 13.4 & \\
\hline Alcohol intake & 18 & 7.32 & \\
\hline $\mathrm{BMI}, \mathrm{kg} / \mathrm{m}^{2}$ & & & $22.32(15.78,46.08)$ \\
\hline$<18.5$ & 27 & 11.0 & \\
\hline $18.5-23.9$ & 138 & 56.1 & \\
\hline $24.0-27.9$ & 70 & 28.5 & \\
\hline$\geq 28$ & 11 & 4.47 & \\
\hline \multicolumn{4}{|l|}{ Gross classification } \\
\hline Elevated & 73 & 29.7 & \\
\hline Ulcerative & 146 & 59.3 & \\
\hline Infiltrative & 27 & 11.0 & \\
\hline \multicolumn{4}{|l|}{ TNM Stage } \\
\hline High-risk II & 73 & 29.7 & \\
\hline III & 173 & 70.3 & \\
\hline $\begin{array}{l}\text { Vascular tumor } \\
\text { thrombus }\end{array}$ & 58 & 23.6 & \\
\hline Perineural invasion & 28 & 11.4 & \\
\hline \multicolumn{4}{|l|}{ Differentiation status } \\
\hline Poor & 51 & 20.7 & \\
\hline High/moderate & 195 & 79.3 & \\
\hline \multicolumn{4}{|l|}{ No. of retrieved } \\
\hline lymph nodes $\geq 12$ & 90 & 36.6 & \\
\hline Intestinal obstruction & 18 & 7.3 & \\
\hline $\mathrm{TG}, \mathrm{mmol} / \mathrm{l}$ & & & $1.03(0.39,4.77)$ \\
\hline $\begin{array}{l}\text { Men } \geq 1.53, \\
\text { women } \geq 1.58\end{array}$ & 36 & 14.6 & \\
\hline APO-B, mmol/l & & & $0.92(0.4,1.86)$ \\
\hline APO-B $\geq 0.73$ & 206 & 83.7 & \\
\hline $\mathrm{CEA}, \mathrm{ng} / \mathrm{ml}$ & & & $3.92(0.203,266.1)$ \\
\hline $\mathrm{CEA} \geq 5.0$ & 102 & 41.5 & \\
\hline \multicolumn{4}{|l|}{ Tumor location } \\
\hline Right-sided colon & 44 & 17.89 & \\
\hline Left-sided colon & 65 & 26.42 & \\
\hline Rectum & 137 & 55.69 & \\
\hline
\end{tabular}

DM, diabetes mellitus; HP, hypertension; BMI, body mass index; TG, triglyceride; APO-B, apolipoprotein-B; CEA, carcinoembryonic antigen.

Epidemiological studies on the association between serum TG levels and cancer prognosis are sparse. Although some studies have assessed the relationship between serum TG levels and prognosis in tumors such as CRC, breast and prostate cancer, the results are inconsistent. In breast cancer, high preoperative serum TG levels were identified 


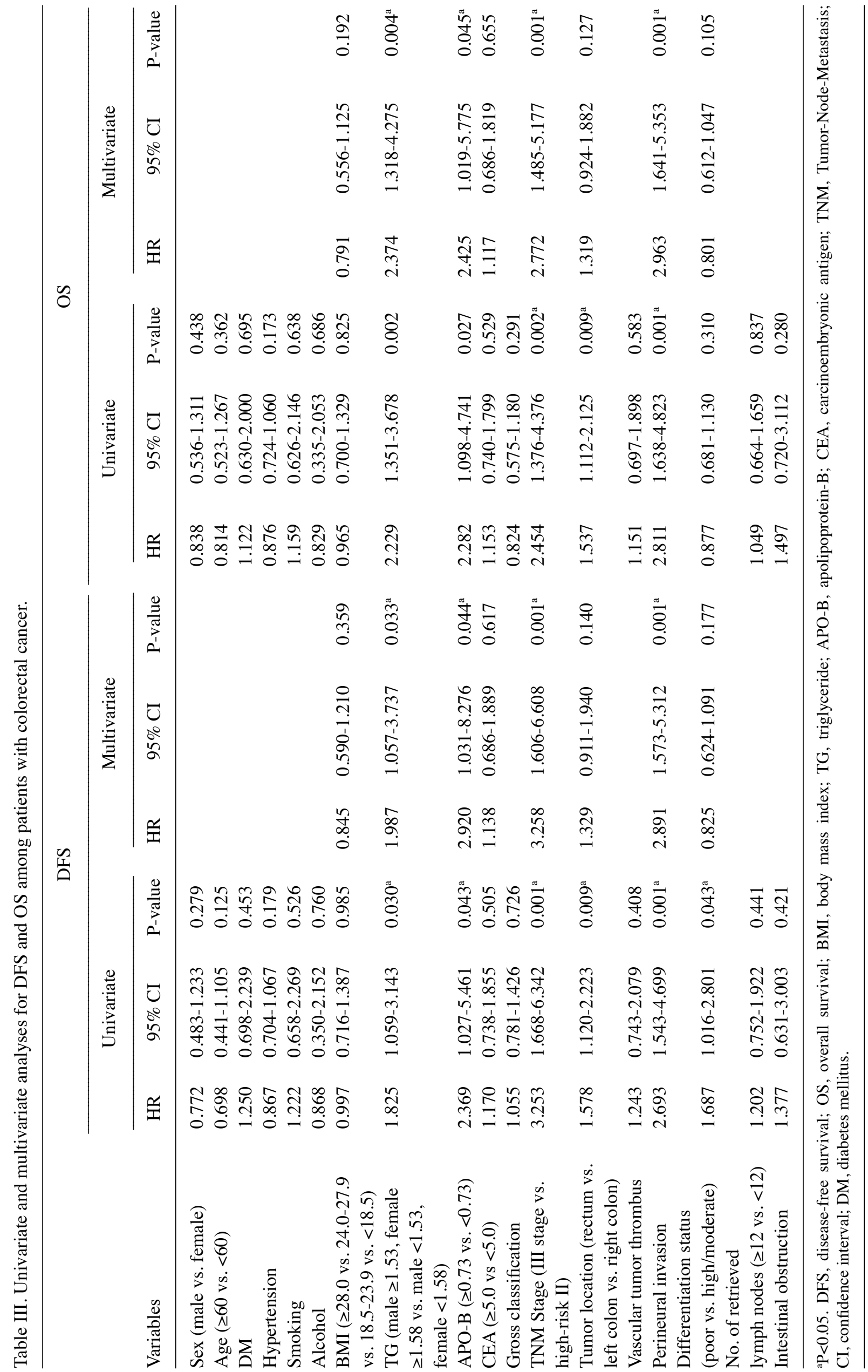


A

Points

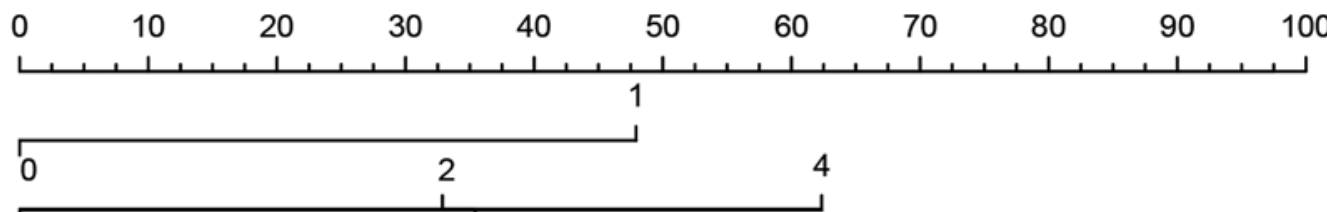

BMI

CEA

TL

Stage

TG

APOB

$\mathrm{Pl}$

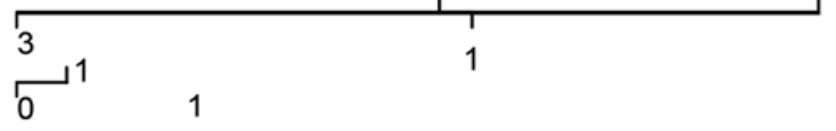

Total points

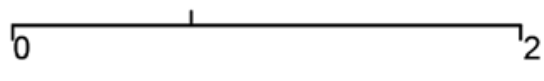

1-Year survival probability
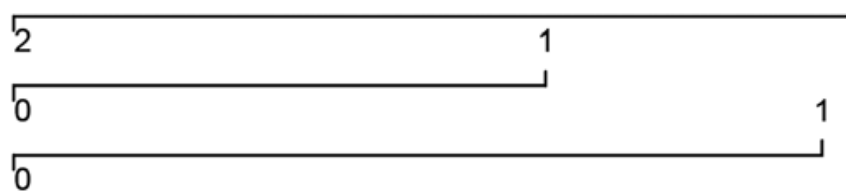

3-Year survival probability

0

5-Year survival probability
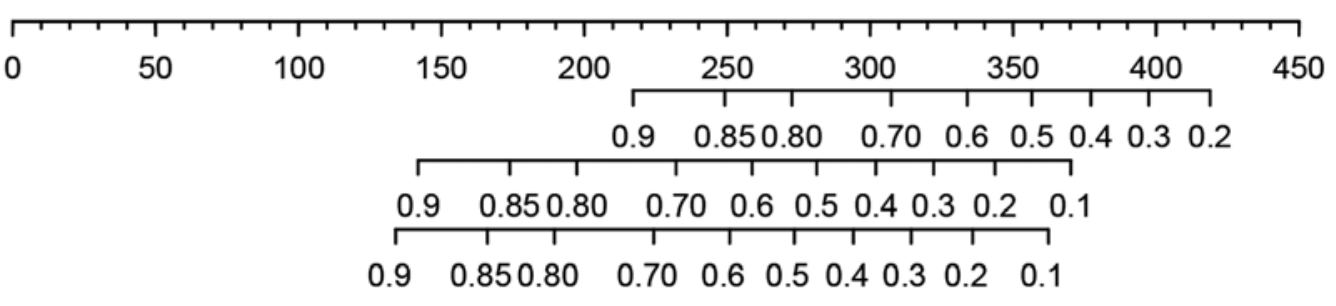

B

Points

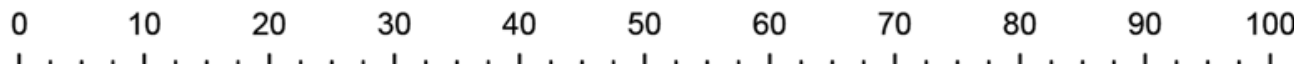

DT

BMI

CEA

TL

Stage

TG

APOB

$\mathrm{Pl}$

Total points

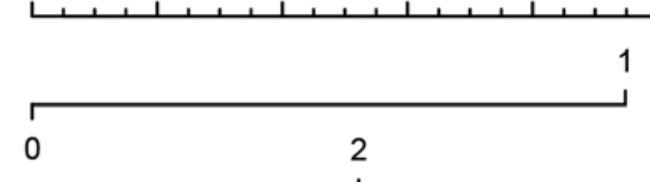

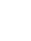


as a significant independent predictor of improved DFS (35). However, in prostate cancer, patients with high TG levels may be at a higher risk of developing aggressive prostate cancer compared with those with low TG levels (36). The association between TG levels and CRC prognosis remains unclear. In a large retrospective study, high TG levels were independently associated with improved OS and recurrence-free survival rates in patients with colon cancer (12). In another study, TG levels were not associated with progression-free survival in patients with colon cancer (37).

Differences in the study population, follow-up time, endpoints and statistical adjustment for confounding factors may have resulted in the conflicting results in the aforementioned studies. In addition, the blood lipid cut-off points were different among previous studies, and these studies did not exclude patients who were using lipid-lowering drugs. To minimize the impact of confounding factors on outcomes, the present study selected patients with stage III and high-risk stage II CRC who had a higher risk of recurrence compared with other patients with non-metastatic CRC who were followed up for a longer period of time. In addition, the present study eliminated the possible bias that could have been introduced by lipid-lowering drugs by excluding patients receiving these medications. The measurements of blood lipid levels were performed under conditions where patients were not allowed to eat a high-fat diet following hospitalization, which ensured the accuracy of these measurements. Considering that blood lipid composition and metabolism in patients with CRC are different from those in healthy individuals (38), the prognostic significance of the established cut-off values was evaluated using a maximal $x^{2}$ method instead of using conventional cut-off values. The maximal $x^{2}$ method can define a subset with the greatest survival difference (39). In the present study, the cut-off values of TG and APO-B were lower compared with the conventional cut-off values, which may be due to the influence of cancer on the metabolic indicators and nutritional status of patients. To the best of our knowledge, this is the first time such an approach has been used in a study to determine the prognostic impact of lipid cut-off points in CRC. After controlling for confounding factors, the present study demonstrated that high TG levels were associated with a $9.87 \%$ greater fully adjusted risk for cancer recurrence. However, Kaplan-Meier survival curves demonstrated that mortality in the low TG group was high in the first 20 months after surgery. This increase in mortality may be attributable to cardiovascular events. Mortality caused by cardiovascular events tends to increase with the increase in TG levels (40), which reduces the proportion of cancer-related deaths in the high TG group. The impaired exercise capacity after surgery has adverse effects on heart function in patients (41); therefore, the risk of cardiovascular complications may be higher during the early postoperative period.

The potential mechanisms for the association between hypertriglyceridemia and cancer development are as follows: i) Increased chronic inflammatory response in patients with hypertriglyceridemia can stimulate cancer cell growth (42); ii) TG levels are positively associated with fecal bile acids, which have been demonstrated to be cytotoxic; this may cause DNA damage and promote CRC progression (43); iii) the results of nutritional studies have demonstrated that a high-fat diet was associated with accelerated tumor growth and metastasis (44); and iv) there is experimental evidence that fatty acid synthase, an enzyme that synthesizes fatty acids, affects tumorigenesis $(45,46)$.

BMI is another important metabolic index; the present study reported that patients with an overweight BMI classification had a favorable prognosis compared with that of patients with a normal or obese BMI classification, which was consistent with the results demonstrated in other studies $(47,48)$.

The present study had some limitations, including its retrospective design. In addition, dietary fat intake (as the percentage of total calories) and metabolic changes during follow-up could not be assessed; therefore, the impact of these factors on prognosis were not analyzed in this study. Since the lipid-specific genetic risk score was not analyzed, it was not possible to evaluate the role of genetic factors in the association between lipid levels and CRC prognosis. Lastly, since this was a single-center study with a small sample size, the possibility of selection bias could not be completely eliminated.

In conclusion, TG and APO-B levels at diagnosis may be independent prognostic factors for stage III and high-risk stage II CRC. Adjusting these parameters at diagnosis to appropriate levels may be beneficial to patients with CRC and may also enable physicians to choose appropriate treatment regimens for these patients. The present study makes a significant contribution to the identification of biomarkers that may be used to accurately predict CRC prognosis, and both TG and APO-B levels can be detected through a low-cost, convenient method. Further prospective, multi-center studies are needed to investigate the exact mechanisms of the association between these biomarkers and CRC prognosis and their biological significance.

\section{Acknowledgements}

The abstract of the present study has been previously published as part of a poster presentation at the 23rd Scientific Meeting of the Chinese Diabetes Society held in Xiamen, Fujian Province, China on November 20-23, 2019.

\section{Funding}

This study was supported by Grants from The National Natural Science Foundation of China (grant no. 81870572); The Medical Innovation Fund Project of Fujian Province (grant no. 2018-CX-23) and The Education and Scientific Research Projects of Young and Middle-aged Teachers in Fujian Province (grant no. JAT190207).

\section{Availability of data and materials}

All datasets used during the present study are available from the corresponding author on reasonable request.

\section{Authors' contributions}

LYY, XMS and SJY conceived and designed the study. XQC and PWW obtained and analyzed the data and edited the drafts. PWW and DHL reviewed the results and participated in the discussion of the data. DHL performed the statistical analysis. XQC and XMS wrote the manuscript. All the authors read and approved the final manuscript. 


\section{Ethics approval and consent to participate}

The present study was approved by the Ethics Committee of the First Affiliated Hospital of Fujian Medical University (approval no. MRCTA, ECFAH of FMU [2019] 200). Written informed consent for data collection and analysis was obtained from the respective patients.

\section{Patient consent for publication}

Not applicable.

\section{Competing interests}

The authors declare that they have no competing interests.

\section{References}

1. Bray F, Ferlay J, Soerjomataram I, Siegel RL, Torre LA and Jemal A: Global cancer statistics 2018: GLOBOCAN estimates of incidence and mortality worldwide for 36 cancers in 185 countries. CA Cancer J Clin 68: 394-424, 2018.

2. O'Connell JB, Maggard MA and Ko CY: Colon cancer survival rates with the new American joint committee on cancer sixth edition staging. J Natl Cancer Inst 96: 1420-1425, 2004.

3. Hari DM, Leung AM, Lee JH, Sim MS, Vuong B, Chiu CG and Bilchik AJ: AJCC cancer staging manual 7th edition criteria for colon cancer: Do the complex modifications improve prognostic assessment? J Am Coll Surg 217: 181-190, 2013.

4. Cerottini JP, Caplin S, Pampallona S and Givel JC: Prognostic factors in colorectal cancer. Oncol Rep 6: 409-414, 1999.

5. Micu BV, Vesa SC, Pop TR and Micu CM: Evaluation of prognostic factors for 5 year-survival after surgery for colorectal cancer. Ann Ital Chir 91: 41-48, 2020.

6. Rashtak S, Ruan X, Druliner BR, Liu H, Therneau T, Mouchli M and Boardman LA: Peripheral neutrophil to lymphocyte ratio improves prognostication in colon cancer. Clin Colorectal Cancer 16: 115-123 e3, 2017.

7. Ma C, Xie J, Luo C, Yin H, Li R, Wang X, Xiong W, Zhang T, Jiang P, Qi W, et al: OxLDL promotes lymphangiogenesis and lymphatic metastasis in gastric cancer by upregulating VEGFC expression and secretion. Int J Oncol 54: 572-584, 2019.

8. Nowak C and Ärnlöv J: A Mendelian randomization study of the effects of blood lipids on breast cancer risk. Nat Commun 9: 3957,2018

9. Murtola TJ, Kasurinen TVJ, Talala K, Taari K, Tammela TLJ and Auvinen A: Serum cholesterol and prostate cancer risk in the Finnish randomized study of screening for prostate cancer. Prostate Cancer Prostatic Dis 22: 66-76, 2019.

10. Raju K, Punnayanapalya SS, Mariyappa N, Eshwarappa SM, Anjaneya $C$ and Kai LJ: Significance of the plasma lipid profile in cases of carcinoma of cervix: A tertiary hospital based study. Asian Pac J Cancer Prev 15: 3779-3784, 2014.

11. Fan TWM, Zhang X, Wang C, Yang Y, Kang WY, Arnold S, Higashi RM, Liu J and Lane AN: Exosomal lipids for classifying early and late stage non-small cell lung cancer. Anal Chim Acta 1037: 256-264, 2018.

12. Yang Y, Mauldin PD, Ebeling M, Hulsey TC, Liu B, Thomas MB, Camp ER and Esnaola NF: Effect of metabolic syndrome and its components on recurrence and survival in colon cancer patients. Cancer 119: 1512-1520, 2013.

13. Peng F, Hu D, Lin X, Chen G, Liang B, Chen Y, Li C, Zhang H, $\mathrm{Xia} Y, \mathrm{Lin} \mathrm{J}$, et al: An in-depth prognostic analysis of baseline blood lipids in predicting postoperative colorectal cancer mortality: The FIESTA study. Cancer Epidemiol 52: 148-157, 2018.

14. Manfredi S, Bouvier AM, Lepage C, Hatem C, Dancourt V and Faivre J: Incidence and patterns of recurrence after resection for cure of colonic cancer in a well defined population. Br J Surg 93: 1115-1122, 2006

15. Benson AB III, Schrag D, Somerfield MR, Cohen AM, Figueredo AT, Flynn PJ, Krzyzanowska MK, Maroun J, McAllister P, Van Cutsem E, et al: American society of clinical oncology recommendations on adjuvant chemotherapy for stage II colon cancer. J Clin Oncol 22: 3408-3419, 2004.
16. O'Connor ES, Greenblatt DY, LoConte NK, Gangnon RE, Liou JI, Heise CP and Smith MA: Adjuvant chemotherapy for stage II colon cancer with poor prognostic features. J Clin Oncol 29: 3381-3388, 2011.

17. Edge SB, Byrd DR, Compton CC, Fritz AG, Greene FL and Trotti A: AJCC cancer staging manual, 7th ed. Springer, New York, NY, 2010.

18. Bosman FT, Carneiro F, Hruban RH and Theise ND: WHO classification of tumours of the digestic system, 4th ed. Lyon: IARC press, 2010.

19. Zhou BF and Cooperative Meta-Analysis Group of the Working Group on Obesity in China: Predictive values of body mass index and waist circumference for risk factors of certain related diseases in Chinese adults-study on optimal cut-off points of body mass index and waist circumference in Chinese adults. Biomed Environ Sci 15: 83-96, 2002.

20. Torsten $\mathrm{H}$ and Berthold L: On the exact distribution of maximally selected rank statistics. Comput Stat Data Anal 43: 121-137, 2003.

21. Brown MS and Goldstein JL: A receptor-mediated pathway for cholesterol homeostasis. Science 232: 34-47, 1986.

22. Kane JP: Apolipoprotein B: Structural and metabolic heterogeneity. Annu Rev Physiol 45: 637-650, 1983.

23. Borgquist S, Butt T, Almgren P, Shiffman D, Stocks T, Orho-Melander M, Manjer J and Melander O: Apolipoproteins, lipids and risk of cancer. Int J Cancer 138: 2648-2656, 2016.

24. Katzke VA, Sookthai D, Johnson T, Kühn T and Kaaks R: Blood lipids and lipoproteins in relation to incidence and mortality risks for CVD and cancer in the prospective EPIC-Heidelberg cohort. BMC Med 15: 218, 2017.

25. Chen S, Lai Y, He Z, Li J, He X, Shen R, Ding Q, Chen H, Peng S and Liu W: Establishment and validation of a predictive nomogram model for non-small cell lung cancer patients with chronic hepatitis B viral infection. J Transl Med 16: 116, 2018.

26. Ma MZ, Yuan SQ, Chen YM and Zhou ZW: Preoperative apolipoprotein B/apolipoprotein A1 ratio: A novel prognostic factor for gastric cancer. Onco Targets Ther 11: 2169-2176, 2018

27. National Cholesterol Education Program (NCEP) Expert Panel on Detection, Evaluation, and Treatment of High Blood Cholesterol in Adults (Adult Treatment Panel III): Third report of the national cholesterol education program (NCEP) expert panel on detection, evaluation, and treatment of high blood cholesterol in adults (adult treatment panel III) final report. Circulation 106: 3143-3421, 2002.

28. Angelin B, Eriksson M and Rudling M: Bile acids and lipoprotein metabolism: A renaissance for bile acids in the post-statin era? Curr Opin Lipidol 10: 269-274, 1999.

29. Fasshauer M and Paschke R: Regulation of adipocytokines and insulin resistance. Diabetologia 46: 1594-1603, 2003.

30. Croft B, Reed M, Patrick C, Kovacevich N and Voutsadakis IA: Diabetes, obesity, and the metabolic syndrome as prognostic factors in stages I to III colorectal cancer patients. J Gastrointest Cancer 50: 221-229, 2019.

31. Shen Z, Ye Y, Bin L, Yin M, Yang X, Jiang K and Wang S: Metabolic syndrome is an important factor for the evolution of prognosis of colorectal cancer: Survival, recurrence, and liver metastasis. Am J Surg 200: 59-63, 2010.

32. Melvin JC, Holmberg L, Rohrmann S, Loda $M$ and Van Hemelrijck M: Serum lipid profiles and cancer risk in the context of obesity: Four meta-analyses. J Cancer Epidemiol 2013: 823849, 2013.

33. Esposito K, Chiodini P, Capuano A, Bellastella G, Maiorino MI, Rafaniello C, Panagiotakos DB and Giugliano D: Colorectal cancer association with metabolic syndrome and its components: A systematic review with meta-analysis. Endocrine 44: 634-647, 2013.

34. Borena W, Stocks T, Jonsson H, Strohmaier S, Nagel G, Bjørge T, Manjer J, Hallmans G, Selmer R, Almquist M, et al: Serum triglycerides and cancer risk in the metabolic syndrome and cancer (Me-Can) collaborative study. Cancer Causes Control 22: 291-299, 2011.

35. Li X, Tang H, Wang J, Xie X, Liu P, Kong Y, Ye F, Shuang Z, $\mathrm{Xie} Z$ and $\mathrm{Xie} X$ : The effect of preoperative serum triglycerides and high-density lipoprotein-cholesterol levels on the prognosis of breast cancer. Breast 32: 1-6, 2017.

36. Hayashi N, Matsushima M, Yamamoto T, Sasaki H, Takahashi $H$ and Egawa S: The impact of hypertriglyceridemia on prostate cancer development in patients aged $\geq 60$ years. BJU Int 109: 515-519, 2012. 
37. Stocks T, Lukanova A, Bjørge T, Ulmer H, Manjer J, Almquist M, Concin H, Engeland A, Hallmans G, Nagel G, et al: Metabolic factors and the risk of colorectal cancer in 580,000 men and women in the metabolic syndrome and cancer project (Me-Can). Cancer 117: 2398-2407, 2011.

38. Pakiet A, Kobiela J, Stepnowski P, Sledzinski T and Mika A: Changes in lipids composition and metabolism in colorectal cancer: A review. Lipids Health Dis 18: 29, 2019.

39. Lausen B, Hothorn T, Bretz F and Schumacher M: Assessment of optimal selected prognostic factors. Biom J 46: 364-374, 2004.

40. Ridker PM: Fasting versus nonfasting triglycerides and the prediction of cardiovascular risk: Do we need to revisit the oral triglyceride tolerance test? Clin Chem 54: 11-13, 2008.

41. Cramer L, Hildebrandt B, Kung T, Wichmann K, Springer J, Doehner W, Sandek A, Valentova M, Stojakovic T, Scharnagl H, et al: Cardiovascular function and predictors of exercise capacity in patients with colorectal cancer. J Am Coll Cardiol 64: 1310-1319, 2014.

42. Kim MH, Kim HN and Choi WS: The association between subclinical inflammation and abnormal glucose and lipid metabolisms in normal-weight Korean individuals. Nutr Metab Cardiovasc Dis 28: 1106-1113, 2018.

43. McKeown-Eyssen G: Epidemiology of colorectal cancer revisited: Are serum triglycerides and/or plasma glucose associated with risk? Cancer Epidemiol Biomarkers Prev 3: 687-695, 1994.
44. Rossi S, Graner E, Febbo P, Weinstein L, Bhattacharya N, Onody T, Bubley G, Balk S and Loda M: Fatty acid synthase expression defines distinct molecular signatures in prostate cancer. Mol Cancer Res 1: 707-715, 2003.

45. Flavin R, Zadra G and Loda M: Metabolic alterations and targeted therapies in prostate cancer. J Pathol 223: 283-294, 2011.

46. Migita T, Ruiz S, Fornari A, Fiorentino M, Priolo C, Zadra G, Inazuka F, Grisanzio C, Palescandolo E, Shin E, et al: Fatty acid synthase: A metabolic enzyme and candidate oncogene in prostate cancer. J Natl Cancer Inst 101: 519-532, 2009.

47. Aparicio T, Ducreux M, Faroux R, Barbier E, Manfredi S, Lecomte T, Etienne PL, Bedenne L, Bennouna J, Phelip JM, et al: Overweight is associated to a better prognosis in metastatic colorectal cancer: A pooled analysis of FFCD trials. Eur J Cancer 98: 1-9, 2018.

48. Lee J, Meyerhardt JA, Giovannucci E and Jeon JY: Association between body mass index and prognosis of colorectal cancer: A meta-analysis of prospective cohort studies. PLoS One 10: e0120706, 2015.

This work is licensed under a Creative Commons Attribution-NonCommercial-NoDerivatives 4.0 International (CC BY-NC-ND 4.0) License. 